\title{
Multi-objective optimal operation of hybrid AC/DC microgrid considering source-network-load coordination
}

\author{
Peng LI ${ }^{1}$, Miaomiao ZHENG $^{1}$ (i)
}

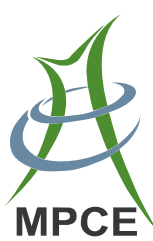

\begin{abstract}
Taking the consumption rate of renewable energy and the operation cost of hybrid AC/DC microgrid as the optimization objectives, the adjustment of load demand curves is carried out considering the demand side response (DSR) on the load side. The complementary utilization of renewable energy between AC area and DC area is achieved to meet the load demand on the source side. In the network side, the hybrid AC/DC microgrids purchase electricity from the power grid at the time-of-use (TOU) price and sell the surplus power of renewable energy to the power grid for profits. The improved memetic algorithm (IMA) is introduced and applied to solve the established mathematical model. The promotion effect of the proposed source-network-load coordination strategies on the optimal operation of hybrid AC/DC microgrid is verified.
\end{abstract}

Keywords Source-network-load coordination, Optimal operation, Improved memetic algorithm, Hybrid AC/DC microgrid, Complementary utilization

CrossCheck date: 8 March 2019

Received: 29 June 2018/ Accepted: 8 March 2019/Published online: 10 June 2019

(c) The Author(s) 2019

$\triangle$ Miaomiao ZHENG

1614299281@qq.com

Peng LI

ncepulp@ncepu.edu.cn

1 School of Electrical and Electronic Engineering, North China Electric Power University, Baoding 071003, China

\section{Introduction}

With the increase of energy crisis and environmental pollution, renewable energy has become the focus of current research because of its inexhaustible characteristics and environmental friendliness [1-3]. However, the output power of renewable energy has significant randomness and intermittency [4]. With the increasing penetration of renewable energy in the power grid, the problems such as the voltage fluctuation, the unreliability of power supply (PS), and the inadequate utilization cannot be ignored $[5,6]$.

It is showed that the coordination among source, network, load and storage can effectively improve the consumption rate of renewable energy and reduce the operation cost and network loss of the grid [7]. The operation economy and the comfort level of occupants are considered at the same time for a residential building through the synergetic optimized dispatch of sources, loads, and storage devices in [8]. It is found that the coordination of energy storage systems, incentive-based and price-based demand responses has a significant effect on the economy of microgrid in [9]. Demand side response (DSR) can increase the consumption rate of renewable energy through load adjustment, and achieve a winwin situation between the power grid and the consumers [10-13]. Considering the uncertainty of wind energy, the effects of allowing large, price-responsive consumers to provide reserves in a power system with high penetration of wind energy are discussed in [14].

Microgrid includes distributed generations (DGs), energy storage (ES) devices, loads, together with power electronic devices, which can realize the efficient utilization of renewable energy through the unified optimized dispatch of DGs [15-18]. The optimal operation of 
microgrid is the focus and difficulty of current research [19-21]. Taking the operation cost of microgrid as the optimization objective, the chaotic binary particle swarm optimization is adopted to solve the established mathematical model in [22]. Based on the chance constrained programming, a new probabilistic optimization framework is developed to optimize the operation cost of microgrid in [23]. A hierarchical optimization strategy is proposed to improve the automation level of DGs in [24]. Hybrid AC/ DC microgrid integrates the advantages of AC microgrid and DC microgrid and its power supply forms are more flexible and diverse, which can satisfy various load demands at the same time. The optimization problem of hybrid AC/DC microgrid has become a research hotspot and difficulty in related fields [25-27].

Current research mainly takes the efficient utilization of renewable energy or economic operation of hybrid AC/DC microgrid as the optimal objectives. In this paper, these two optimal objectives are both considered and a multi-objective optimal operation method of hybrid AC/DC microgrid considering source-network-load coordination is proposed. The mathematical model of hybrid AC/DC microgrid is a multi-objective, multi-constraint and nonlinear model which needs a good solving method. Finding a new algorithm or improving the existing algorithms is one of the research topics currently [28-30]. An algorithm based on the iterative reweighted $l_{1}$-norm approximation is developed in [31] that involves solving a sequence of semidefinite programs. The simulation shows that the algorithm has a shorter running time and returns a solution with a smaller gap from the global optimal solution. In order to solve the optimal power routing problem in clusters of DC microgrids, a novel cloud-based approach is proposed and its effectiveness has been verified in [32]. Memetic algorithm (MA) has the double search mechanisms, i.e., local search and global search. Compared with the conventional algorithms, MA has a significant advantage in the convergence precision [33], which is adopted in this paper. To sum up, the main contributions of this paper are as follows.

1) Under the promise of meeting the constraint of customer satisfaction, the incentive-based DSR and price-based DSR are both considered on the load side. Firstly, the load is shifted to close to the output power curve of renewable energy considering the incentivebased DSR. Next, according to the time-of-use (TOU) price of the power grid, the price-based DSR is considered in the order of the peak price time to the valley price time to optimize the operation costs of hybrid AC/DC microgrid. It can improve the consumption rate of renewable energy and reduce the operation costs of hybrid AC/DC microgrid to a large extent.
2) On the source side, the renewable energy is given priority to supply the load whose generation cost is not considered. While the output power of renewable energy in one area has not reached the upper limitation and the load demand in the other area is not satisfied, the complementary utilization of renewable energy is achieved between AC area and DC area. On the network side, under the premise of meeting the capacity constraint of the point of common coupling (PCC), the hybrid AC/DC microgrids can purchase the electricity from the power grid at the TOU price. When the load demands of AC area and DC area are both satisfied, the surplus power of renewable energy can be sold to the power grid if the unit generation cost of renewable energy is smaller than the selling price, which can achieve a win-win situation between the power grid and hybrid AC/DC microgrids.

3) In view of the existing disadvantages of the basic MA, MA is improved in the process of initialization, distribution of the ordinary individuals, local search and global search. The improved MA (IMA) is applied to solve the established mathematical model. The correctness and effectiveness of the proposed optimal operation method and the IMA are verified through a practical case. The simulation results show that the convergence time is only $10.22 \mathrm{~s}$ and the IMA can find the optimal solution quickly and accurately. The consumption rate of renewable energy can reach up to $100 \%$. Comparing with the calculation results of hybrid AC/DC microgrids without considering sourcenetwork-load coordination, the cost of hybrid AC/DC microgrids decreases from $\$ 1533.7$ to $\$ 1442.4$, which further proves the feasibility of the IMA.

\section{Mathematical model of optimal operation}

\subsection{Source-network-load coordination}

\subsubsection{Load side}

1) Shift load for consuming renewable energy

In order to avoid the abandonment of renewable energy, the load demand curves would be adjusted according to the prediction data of renewable energy considering the DSR based on the incentive and the price. The value of shift-in load is determined by the difference between the output power of renewable energy and the load demand. The specific process is shown as follows: 


$$
\begin{aligned}
& L_{\mathrm{yr}}(t)= \begin{cases}P_{\mathrm{r}}(t)-L(t) & P_{\mathrm{r}}(t)>L(t) \\
0 & P_{\mathrm{r}}(t) \leq L(t)\end{cases} \\
& L_{\mathrm{ycl}}(t)= \begin{cases}\min \left(L_{\mathrm{py}}(t),\right. & \left.\sum_{t=1}^{T} L_{\mathrm{yr}}(t)-\sum_{t t=0}^{t-1} L_{\mathrm{ycl}}(t t)\right) L(t)>P_{\mathrm{r}}(t) \\
0 & L(t) \leq P_{\mathrm{r}}(t)\end{cases}
\end{aligned}
$$$$
L Y R(t)= \begin{cases}P_{\mathrm{r}}(t) & P_{\mathrm{r}}(t)>L(t) \\ 0 & P_{\mathrm{r}}(t) \leq L(t)\end{cases}
$$$$
L_{\mathrm{yrl}}(t)=\min \left(L_{\mathrm{yr}}(t), \quad P_{\mathrm{r}}(t) / \sum_{t=1}^{T} L Y R(t) \times \sum_{t=1}^{T} L_{\mathrm{yc} 1}(t)\right)
$$

$L_{\mathrm{j} 1}(t)= \begin{cases}L(t)-L_{\mathrm{yc} 1}(t) & L(t)>P_{\mathrm{r}}(t) \\ L_{\mathrm{yr} 1}(t)+L(t) & L(t) \leq P_{\mathrm{r}}(t)\end{cases}$

where $L(t)$ is the initial value of load demand; $L_{\mathrm{yr}}(t)$ is the maximum shift-in load at time $t ; L_{\mathrm{ycl}}(t)$ and $L_{\mathrm{yr} 1}(t)$ is the shift-out load and the shift-in load at time $t, L_{\mathrm{ycl}}(0)=0$; $L_{\mathrm{py}}(t)$ is the shiftable load at time $t ; P_{\mathrm{r}}(t)$ is the predictive output power of renewable energy at time $t ; T$ is the optimal cycle; $L Y R(t)$ is the total power of renewable energy while the its output power is bigger than the load; and $L_{\mathrm{jl}}(t)$ is the value of load at time $t$ after shifting the load.

2) Shift load for reducing running cost of hybrid AC/DC microgrid

In order to reduce the cost of hybrid AC/DC microgrid, the load curves would be adjusted considering the DSR based on price. Under the promise of guaranteeing the consumption rate of renewable energy and meeting the constraints of the supply-load balance and the customer satisfaction degree, the load would be shifted according to the TOU price of the power grid.

$L_{\mathrm{jg}}(t)=L_{\mathrm{j} 1}(t)-L_{\mathrm{yc} 2}(t)+L_{\mathrm{yr} 2}(t)$

where $L_{\mathrm{jg}}(t)$ represents the load demand value at time $t$ considering the DSR based on price; $L_{\mathrm{yc} 2}(t)$ and $L_{\mathrm{yr} 2}(t)$ represent the shift-out load and shift-in load at time $t$.

\subsubsection{Source side}

This paper studies a hybrid AC/DC microgrid which contains wind turbine (WT) and diesel generator (DEG) in $\mathrm{AC}$ area, and photovoltaics (PV) and ES in DC area. In the source side, the renewable energy is given priority to supply the load. When the renewable energy is difficult to meet the load demand, the output of other power sources is determined by the cost functions. The output power of WT and PV is mainly determined by the weather. The statistical data show that the wind speed obeys the Weibull distribution and the intensity of illumination obeys the Beta distribution. Through the complementary power supply of renewable energy between AC area and DC area, it can make up for the problems that the peak output power of WT and PV cannot be completely consumed and the load demand is difficult to meet when the output power of WT and PV is close to 0 , which can effectively increase the consumption rate of renewable energy.

$$
\begin{aligned}
& \text { If }\left(R_{\mathrm{AC}}(t)>L_{\mathrm{AC}}(t)\right) \cap\left(R_{\mathrm{DC}}(t)<L_{\mathrm{DC}}(t)\right), \\
& \left\{\begin{array}{l}
R_{\mathrm{AC} \rightarrow \mathrm{DC}}(t)=\min \left(R_{\mathrm{AC}}(t)-L_{\mathrm{AC}}(t), L_{\mathrm{DC}}(t)-R_{\mathrm{DC}}(t)\right) \\
R_{\mathrm{DC} \rightarrow \mathrm{AC}}(t)=0 \\
P_{\mathrm{RAC}}(t)=L_{\mathrm{AC}}(t) \\
P_{\mathrm{RDC}}(t)=R_{\mathrm{DC}}(t)
\end{array}\right.
\end{aligned}
$$

$$
\text { If }\left(R_{\mathrm{AC}}(t) \geq L_{\mathrm{AC}}(t)\right) \cap\left(R_{\mathrm{DC}}(t) \geq L_{\mathrm{DC}}(t)\right),
$$

$\left\{\begin{array}{l}R_{\mathrm{AC} \rightarrow \mathrm{DC}}(t)=0 \\ R_{\mathrm{DC} \rightarrow \mathrm{AC}}(t)=0 \\ P_{\mathrm{RAC}}(t)=L_{\mathrm{AC}}(t) \\ P_{\mathrm{RDC}}(t)=L_{\mathrm{DC}}(t)\end{array}\right.$

$$
\begin{aligned}
& \text { If }\left(R_{\mathrm{AC}}(t)<L_{\mathrm{AC}}(t)\right) \cap\left(R_{\mathrm{DC}}(t)>L_{\mathrm{DC}}(t)\right), \\
& \left\{\begin{array}{l}
R_{\mathrm{AC} \rightarrow \mathrm{DC}}(t)=0 \\
R_{\mathrm{DC} \rightarrow \mathrm{AC}}(t)=\min \left(L_{\mathrm{AC}}(t)-R_{\mathrm{AC}}(t), R_{\mathrm{DC}}(t)-L_{\mathrm{DC}}(t)\right) \\
P_{\mathrm{RAC}}(t)=R_{\mathrm{AC}}(t) \\
P_{\mathrm{RDC}}(t)=L_{\mathrm{DC}}(t)
\end{array}\right.
\end{aligned}
$$

$$
\text { If }\left(R_{\mathrm{AC}}(t) \leq L_{\mathrm{AC}}(t)\right) \cap\left(R_{\mathrm{DC}}(t) \leq L_{\mathrm{DC}}(t)\right),
$$

$\left\{\begin{array}{l}R_{\mathrm{AC} \rightarrow \mathrm{DC}}(t)=0 \\ R_{\mathrm{DC} \rightarrow \mathrm{AC}}(t)=0 \\ P_{\mathrm{RAC}}(t)=R_{\mathrm{AC}}(t) \\ P_{\mathrm{RDC}}(t)=R_{\mathrm{DC}}(t)\end{array}\right.$

where $R_{\mathrm{AC}}(t)$ and $R_{\mathrm{DC}}(t)$ represent the maximum output power of the renewable energy in AC area and DC area; $L_{\mathrm{AC}}(t)$ and $L_{\mathrm{DC}}(t)$ represent the load in $\mathrm{AC}$ area and $\mathrm{DC}$ area considering the DSR; $R_{\mathrm{AC} \rightarrow \mathrm{DC}}(t)$ represents the output power of renewable energy in $\mathrm{AC}$ area flowing from $\mathrm{AC}$ area to $\mathrm{DC}$ area; $R_{\mathrm{DC} \rightarrow \mathrm{AC}}(t)$ represents the output power of renewable energy in DC area flowing from DC area to AC area; $P_{\mathrm{RAC}}(t)$ represents the output power of the renewable energy in AC area which supplies the load in AC area; and $P_{\mathrm{RDC}}(t)$ represents the output power of the renewable energy in DC area which supplies the load in DC area.

\subsubsection{Network side}

The hybrid AC/DC microgrid in this paper is connected with the power grid which is equivalent to a big power supply for the achievement of supply-load balance. Under the premise of meeting the transmission capacity constraint of the PCC, the hybrid AC/DC microgrid can purchase 
electricity from the power grid at the TOU price, and the cost of purchasing electricity can be calculated as:

$F_{\text {grid }}=\sum_{t=1}^{T} \varepsilon(t) P_{\text {grid }}(t)$

where $F_{\text {grid }}$ represents the costs of purchasing electricity from the power grid; $\varepsilon(t)$ represents the TOU price; and $P_{\text {grid }}(t)$ represents the purchased electricity.

When the internal load demand is met in the hybrid AC/ DC microgrid, the surplus power of renewable energy can be sold to the power grid if the unit generation cost of renewable energy is less than the selling price.

$F_{\mathrm{s}}=\sum_{t=1}^{T}(\beta(t)-\chi(t)) R_{\mathrm{s}}(t)$

where $F_{\mathrm{s}}$ is the profits of selling the surplus power of renewable energy to the power grid; $\beta(t)$ is the selling price at time $t ; \chi(t)$ is the unit generating cost of renewable energy; and $R_{\mathrm{s}}(t)$ is the surplus power sold to the grid.

To sum up, according to the proposed optimal operation method of hybrid AC/DC microgrid, the source-networkload coordination can be shown as Fig. 1.

\subsection{Optimization objectives}

\subsubsection{Operation cost}

1) Initial construction $\operatorname{cost} F_{\mathrm{c}}$ :

$F_{\mathrm{c}}=\sum_{t=1}^{T} \sum_{i=1}^{N} \frac{k_{\mathrm{hs}, i} F_{\mathrm{gd}, i}(t)}{8760}$

where $N$ is the number of power supplies; $k_{\mathrm{hs}, i}$ is the investment coefficient of power supply $i$, which is determined by the service life of the power supply; and $F_{\mathrm{gd}, i}(t)$ is the fixed investment costs of power supply $i$.

2) Operation and maintenance cost $F_{\text {om: }}$ :

$F_{\text {om }}=\sum_{t=1}^{T} \sum_{i=1}^{N} \gamma_{i} P_{i}(t)$

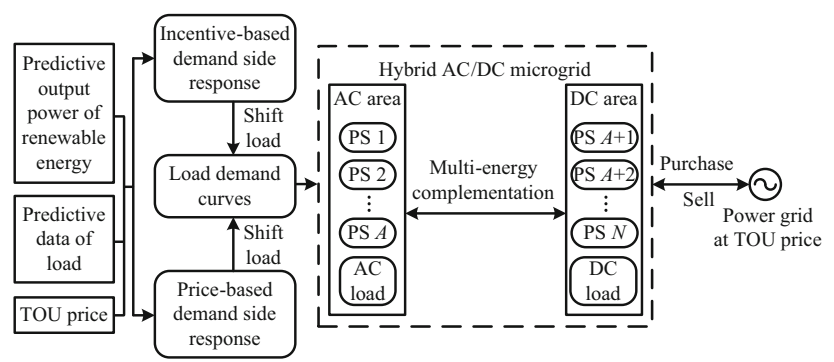

Fig. 1 Source-network-load coordination where $\gamma_{i}$ is the operation and maintenance coefficient of power supply $i$; and $P_{i}(t)$ is the output power of power supply $i$.

3) Fuel cost $F_{\text {fuel }}$ :

$F_{\text {fuel }}=\sum_{t=1}^{T} a+b P_{\mathrm{DEG}}(t)+c P_{\mathrm{DEG}}(t)^{2}$

where $a, b, c$ are the cost coefficients of DEG; and $P_{\mathrm{DEG}}(t)$ is the output power of DEG.

4) Environmental cost $F_{\text {en }}$ :

$F_{\text {en }}=\sum_{t=1}^{T} \tau_{e} E_{e} P_{i}(t)$

where $\tau_{e}$ represents the emission coefficient of the pollution $e$; and $E_{e}$ represents the emission factor of the pollution $e$.

5) Subsidy cost to the customers who adjust the load demand curve $F_{\text {py }}$ :

$F_{\mathrm{py}}=\alpha \sum_{t=1}^{T} \frac{\left|L(t)-L_{\mathrm{jg}}(t)\right|}{2}$

where $\alpha$ represents the subsidy coefficient.

6) Renewable energy generating subsidies $F_{\mathrm{r}}$.

$$
\begin{aligned}
P_{\mathrm{R}}(t)= & P_{\mathrm{RAC}}(t)+P_{\mathrm{RDC}}(t)+R_{\mathrm{AC} \rightarrow \mathrm{DC}}(t) \\
& +R_{\mathrm{DC} \rightarrow \mathrm{AC}}(t)+R_{\mathrm{S}}(t)
\end{aligned}
$$

$F_{\mathrm{r}}=\sum_{t=1}^{T} \delta(t) P_{\mathrm{R}}(t)$

where $P_{\mathrm{R}}(t)$ is the output power of renewable energy; and $\delta(t)$ is the unit generating subsidy price of renewable energy.

In summary, the total costs of hybrid AC/DC microgrid $F$ can be expressed as follows:

$F=F_{\mathrm{c}}+F_{\text {om }}+F_{\text {fuel }}+F_{\text {en }}+F_{\text {grid }}+F_{\text {py }}-F_{\mathrm{r}}-F_{\mathrm{s}}$

\subsubsection{Consumption rate of renewable energy}

$R(t)=R_{\mathrm{AC}}(t)+R_{\mathrm{DC}}(t)$

$K=\frac{\sum_{t=1}^{T} P_{\mathrm{R}}(t)}{\sum_{t=1}^{T} R(t)}$

where $R(t)$ is the maximum output power of renewable energy in the hybrid AC/DC microgrid at time $t$; and $K$ is the 
consumption rate of renewable energy, which is the ratio of the actual output power to the maximum output power of renewable energy in the hybrid AC/DC microgrid.

The operation cost of hybrid AC/DC microgrid and the consumption rate of renewable energy are both the optimal objectives in this paper. The latter has a higher optimal priority which means the operation economy of hybrid AC/DC microgrid would be sacrificed if the consumption rate of renewable energy is influenced. While the related technologies of renewable energy become more and more mature and the consumption rate of renewable energy has met the requirements in the future, the operation economy of hybrid $\mathrm{AC} / \mathrm{DC}$ microgrid can be taken as the main optimization object.

\subsection{Constraints}

1) Supply-load balance constraints:

$$
\left\{\begin{array}{l}
\sum_{t=1}^{T} P_{\mathrm{RAC}}(t)+P_{\mathrm{DEG}}(t)+R_{\mathrm{DC} \rightarrow \mathrm{AC}}(t)+P_{\text {gridA }}(t)=L_{\mathrm{AC}}(t) \\
\sum_{t=1}^{T} P_{\mathrm{RDC}}(t)+P_{\mathrm{ES}}(t)+R_{\mathrm{AC} \rightarrow \mathrm{DC}}(t)+P_{\text {gridD }}(t)=L_{\mathrm{DC}}(t)
\end{array}\right.
$$

2) Capacity constraint of the PCC:

$\left|P_{\mathrm{G}}(t)\right| \leq P_{\mathrm{G}, \max }$

3) Constraints of ES:

$$
\left\{\begin{array}{l}
S O C(t+1)=S O C(t)-\left(\eta_{\mathrm{ES}} P_{\mathrm{ES}}(t)\right) / Q_{\mathrm{ES}, \max }(t) \\
S O C_{\min } \leq S O C(t) \leq S O C_{\max } \\
S O C_{\text {start }}=S O C_{\mathrm{end}}
\end{array}\right.
$$

4) Changing range constraint of each power supply:

$P_{i, \min } \leq P_{i}(t) \leq P_{i, \max }$

5) Constraints of shifting load:

$$
\left\{\begin{array}{l}
\sum_{t=1}^{T} L_{\mathrm{yc} 1}(t)=\sum_{t=1}^{T} L_{\mathrm{yr} 1}(t) \\
\sum_{t=1}^{T} L_{\mathrm{yc} 2}(t)=\sum_{t=1}^{T} L_{\mathrm{yr} 2}(t) \\
\lambda=1-\sum_{t=1}^{T}\left|L(t)-L_{\mathrm{jg}}(t)\right| / \sum_{t=1}^{T} L(t) \quad \lambda \geq \lambda_{\text {min }}
\end{array}\right.
$$

where $P_{\text {gridA }}(t)$ and $P_{\text {gridD }}(t)$ are the electricity purchased from the power grid in AC area and DC area; $P_{\mathrm{G}}(t)$ and $P_{\mathrm{G}, \max }$ are the transmitting power and capacity of the PCC; $S O C(t)$ is the state of charge (SOC) of ES at time $t ; P_{\mathrm{ES}}(t)$ is the output power of ES, which is positive while discharging and is negative while charging; $\eta_{\mathrm{ES}}$ is the charging and discharging efficiency of ES; $Q_{\mathrm{ES} \text {,max }}(t)$ is the capacity of ES; $S O C_{\min }$ and $S O C_{\max }$ are the range of SOC; $S O C_{\text {start }}$ and $S O C_{\text {end }}$ are the initial value and the final value of SOC; $P_{i, \text { min }}$ and $P_{i, \max }$ are the range of output power of the power supply $i ; \lambda$ is the satisfaction degree of customers, which is decreased with the increase of shifting load; and $\lambda_{\min }$ is the lower limitation of $\lambda$.

To sum up, a big picture schematic overview of the paper can be concluded for the sake of clarification. Figure 2 shows the optimization model of hybrid AC/DC microgrid. The IMA for solving the optimization model would be introduced in the next section.

\section{MA}

\subsection{Basic MA}

Pablo Moscato imitated the way of biological evolution in nature and applied it in cultural evolution. In 1989, he first proposed the MA [33]. The feature of MA is that its iterative process can be divided into local search and global search, which guarantees the convergence accuracy. The specific iterative process of MA is shown as follows.

1) Initialization of MA

$x=x_{\min }+\operatorname{rand}(0,1) \times\left(x_{\max }-x_{\min }\right)$

where $x_{\min }$ and $x_{\max }$ are the range of the individual $x$; and $\operatorname{rand}(0,1)$ is a random number which obeys uniform distribution and values in $[0,1]$.

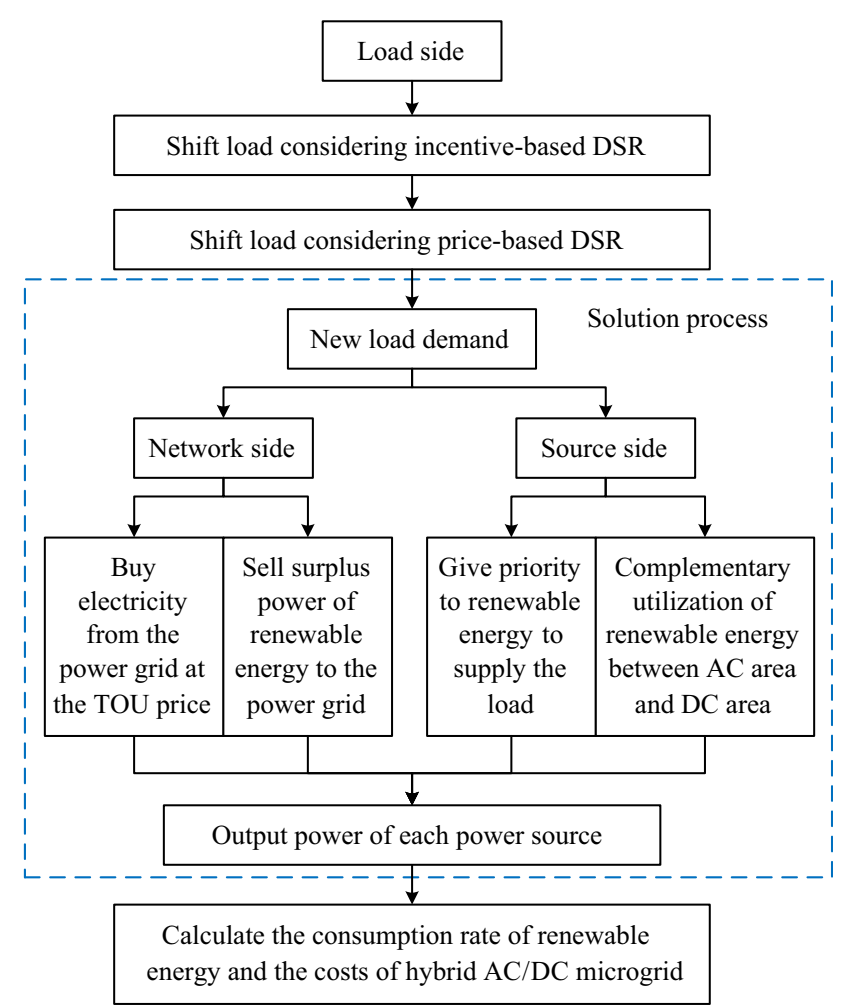

Fig. 2 Optimization model of hybrid AC/DC microgrid 
After the initialization, the population containing $M$ individuals is generated and all initial individuals are sorted according to their fitness values. The individuals from 1 to $M_{\text {agent }}$ in the sequence are the agents. The remaining individuals are the ordinary individuals and the number of the ordinary individuals is $M_{\text {public }}$. All ordinary individuals would be distributed to the agents in the subsequent steps.

\section{2) Distribution of the ordinary individuals}

The number of the ordinary individuals which belong to the agent $a$ is determined by the relative strength and the strength of each agent.

$S_{a}=\max \left\{s_{b}\right\}-s_{a} \quad b=1,2, \cdots, M_{\text {agent }}$

$p_{a}=\frac{S_{a}}{\sum_{a=1}^{M_{\text {agent }}} S_{a}}$

$M_{a, p}=\operatorname{round}\left(p_{a} M_{\text {public }}\right)$

where $s_{a}$ and $s_{b}$ are the fitness values of the agent $a$ and $b$, respectively; $S_{a}$ and $p_{a}$ are the relative strength and the strength of the agent $a$, respectively; and $M_{a, p}$ is the number of the ordinary individuals which belong to the agent $a$.

\section{3) Local search}

In the local search process, the ordinary individuals would move towards their agents.

$x_{b}(D e+1)=x_{b}(D e)+\gamma d$

where $x_{b}(D e)$ is the position of the ordinary individual $b$; $D e$ is the current iteration number; $\gamma$ is the moving coefficient; $d$ is the distance between the ordinary individual $b$ and its agent.

In order to increase the diversity of MA, the population updating is performed in each iteration process. There are $M_{a, p r}$ individuals which need to be regenerated among the ordinary individuals owned by the agent $a$.

$M_{a, p r}=\operatorname{round}\left(q M_{a, p}\right)$

where $q$ is the updating proportion.

\section{4) Global search}

After the local search, it needs to reorder the population and select new agents. In the global search process, the individual with the worst fitness value among all individuals would be taken as the competed goal by each agent. The specific competition process is shown as follows:

$T S_{a}=S_{a}+\xi \frac{\sum_{b=1}^{M_{a, p}} w_{b}}{M_{a, p}}$
$M T S_{a}=\max \left\{T S_{b}\right\}-T S_{a}$

where $T S_{a}$ and $M T S_{a}$ are the total strength and the total relative strength of the agent $a$, respectively; $\xi$ is the weight value of the ordinary individuals; and $w_{b}$ is the fitness value of the ordinary individual $b$ which belongs to the agent $a$.

$c p_{a}=\left|\frac{M T S_{a}}{\sum_{b=1}^{M_{\text {agent }}} M T S_{b}}\right|$

$\boldsymbol{P}=\left[\begin{array}{llll}c p_{1} & c p_{2} & \cdots & c p_{M_{\text {agent }}}\end{array}\right]$

where $c p_{a}$ is the competition probability of the agent $a$; and $\boldsymbol{P}$ is the competition probability vector of all agents.

Let $\boldsymbol{R}$ be a random vector of the same dimension as $\boldsymbol{P}$, and each element of $\boldsymbol{R}$ is valued in [0,1]. The competed individual would be obtained by the agent corresponding to the biggest element in the vector which is the difference between $\boldsymbol{P}$ and $\boldsymbol{R}$.

In order to increase the competitiveness of the agents, they would cooperate with each other. If the distance between any two agents is shorter than the cooperation distance $D$, the two agents would be merged, and all the ordinary individuals of these two agents would belong to the agent with a bigger total strength.

$D=\operatorname{norm}\left(x_{\max }-x_{\min }\right) \times u$

where $u$ is the cooperation coefficient.

\section{5) End of MA}

After the local search and global search, the agent which has no ordinary individuals would be eliminated. When there is only one agent in the algorithm, the agent is the solution of the objective function. MA would stop when the algorithm meets the requirement of convergence accuracy or reaches the maximum number of iterations.

\subsection{IMA}

The specific iterative process of IMA is shown as follows.

1) Initialization of IMA

The initial individuals are generated randomly in MA and the difference between the individuals is uncertain and large, which affects the convergence speed and accuracy. In the IMA, the initialization process would be circulated $O$ times and the total number of individuals is $O \times M$. These individuals are sorted according to their fitness values. The individuals from 1 to $M_{\text {agent }}$ in the sequence are the agents. The individuals from $M_{\text {agent }}+1$ to $M_{\text {public }}$ are the ordinary individuals. The rest individuals of the population would be 
eliminated. It is equivalent to screening a group of superior in the population to improve the convergence performance of the algorithm.

\section{2) Distribution of the ordinary individuals}

According to the process of the distribution of the ordinary individuals, it can be seen that the relative strength $S$ and the strength $p$ of the agent with the best fitness value are 0 in the MA. The number of ordinary individuals belonging to the agent is 0 and the agent would be eliminated, which may influence the convergence accuracy. In this paper, the calculating process of the relative strength is improved as follows:

$S_{a}=\tau \times \max \left\{s_{b}\right\}-s_{a}$

where $\tau$ is a constant which is greater than 1. Experiments show that the algorithm performance is great when the value of $\tau$ is equal to 1.3 .

\section{3) Local search}

In the process of local search, the moving distance of each ordinary individual is the distance between themselves and their agents. Inspired by the particle swarm optimization (PSO) [34], the moving distance is improved that the distance between the ordinary individuals and their agents, and the distance between the ordinary individuals and the agent with the best fitness value among all agents are both considered.

$x_{b}(D e+1)=x_{b}(D e)+r_{1} \gamma_{1} d_{1}+r_{2} \gamma_{2} d_{2}$

where $r_{1}$ and $r_{2}$ are two random numbers which obey uniform distribution and value in $[0,1] ; \gamma_{1}$ and $\gamma_{2}$ are the local moving coefficient and the global moving coefficient; $d_{1}$ is the distance between the ordinary individuals and their agents; and $d_{2}$ is the distance between the ordinary individuals and the agent with the best fitness value among all agents.

\section{4) Global search}

In the MA, the global search is divided into two parts: competition and cooperation. The cooperation coefficient between agents is a constant. After several experiments, it is found that the value of cooperation coefficient has great influence on the convergence speed of the algorithm. Summing up the experimental rule, the cooperation coefficient is concluded as follows:

$u=\sin \left(\frac{\pi}{2} \times \frac{D e}{D e s}\right)$

where Des is the total iteration times.

5) End of IMA

\subsection{Performance test}

In order to test the optimization performance of the IMA, the test function of Rosenbrock is solved by PSO, MA, and IMA, respectively. The function of Rosenbrock is expressed as follows:

$f=\sum_{i=1}^{n}\left[100\left(x_{i+1}-x_{i}^{2}\right)^{2}+\left(x_{i}-1\right)^{2}\right]$

where $n$ is the dimension of variables with a value of 15 .

The variable range is from -30 to 30 . The size of the population is set as 100 and the number of agents is set as 5 . The number of iterations is 1000 . Each algorithm runs 10 times and the average running results are shown in Table 1. The convergence situation of each algorithm is shown as Fig. 3.

From the Table 1 and Fig. 3, it can be seen that IMA and MA have a significant advantage over PSO in the convergence precision. After the improvement in the process of the initialization, the distribution of the ordinary individuals, the local search and the global search, the convergence performance of the algorithm is greatly improved. The convergence accuracy and convergence speed of IMA can be met at the same time, which has ideal optimization performance. It is verified that IMA has the ability to solve the complex optimization problem, which is suitable to solve the multi-objective, multi-constraint, nonlinear mathematical model established in this paper.

\section{Simulation based on engineering data}

In this paper, the correctness and feasibility of the proposed optimal operation method are verified based on the data in a hybrid AC/DC microgrid demonstration project. The topological structure of hybrid AC/DC microgrid is shown in Fig. 4. The parameters of each power supply are listed in Table 2.

WT is connected to the AC bus through AC/AC convertor. PV is connected to the DC bus through DC/DC convertor. The voltage of WT and PV is changed through the transformers to the same as the voltage level of bus. WT is coupled to the doubly-fed induction generator. TOU price is listed in Appendix A Table A1. Investment

Table 1 Running results of each algorithm

\begin{tabular}{llc}
\hline Algorithm & Average result & Running time (s) \\
\hline PSO & 6.6207 & 17.287467 \\
MA & 2.2513 & 4.544177 \\
IMA & 1.4265 & 1.332475 \\
\hline
\end{tabular}




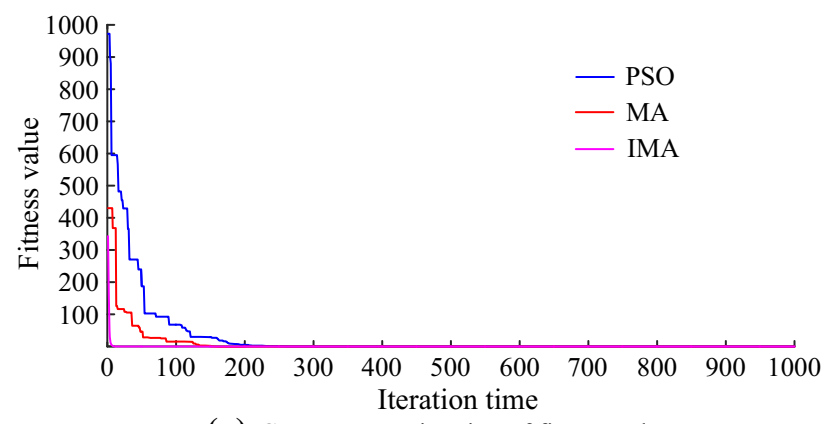

(a) Convergence situation of fitness value

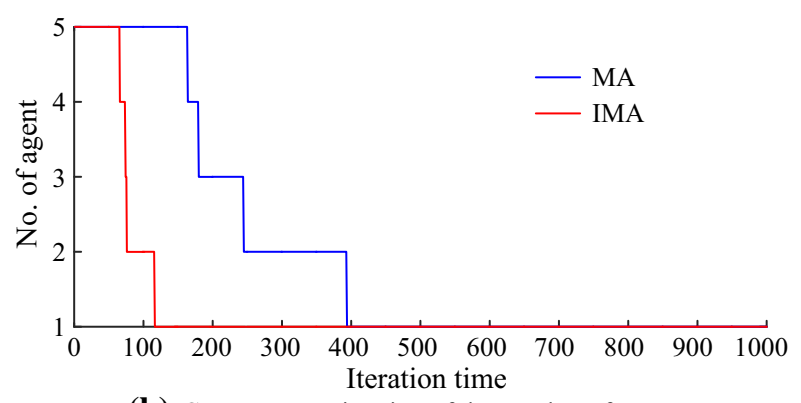

(b) Convergence situation of the number of agents

Fig. 3 Convergence situation of each algorithm

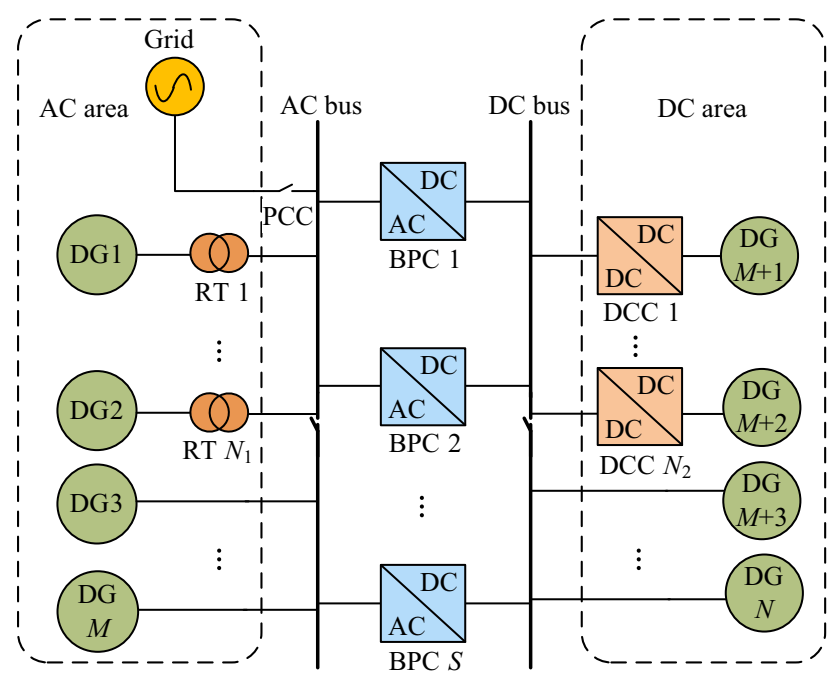

Fig. 4 Topological structure of hybrid AC/DC microgrid

Table 2 Parameters of power supplies

\begin{tabular}{llll}
\hline $\begin{array}{l}\text { Supply } \\
\text { area }\end{array}$ & $\begin{array}{l}\text { Power } \\
\text { supply }\end{array}$ & Capacity & $\begin{array}{l}\text { Operation and maintenance } \\
\text { coefficient }(\$ / \mathrm{kWh})\end{array}$ \\
\hline AC & WT & $2 \mathrm{MW}$ & 0.0044 \\
& DEG & $700 \mathrm{~kW}$ & 0.0129 \\
DC & PV & $2 \mathrm{MW}$ & 0.0014 \\
& ES & $250 \mathrm{~kW} /$ & 0.0013 \\
& & $1 \mathrm{MWh}$ & \\
\hline
\end{tabular}

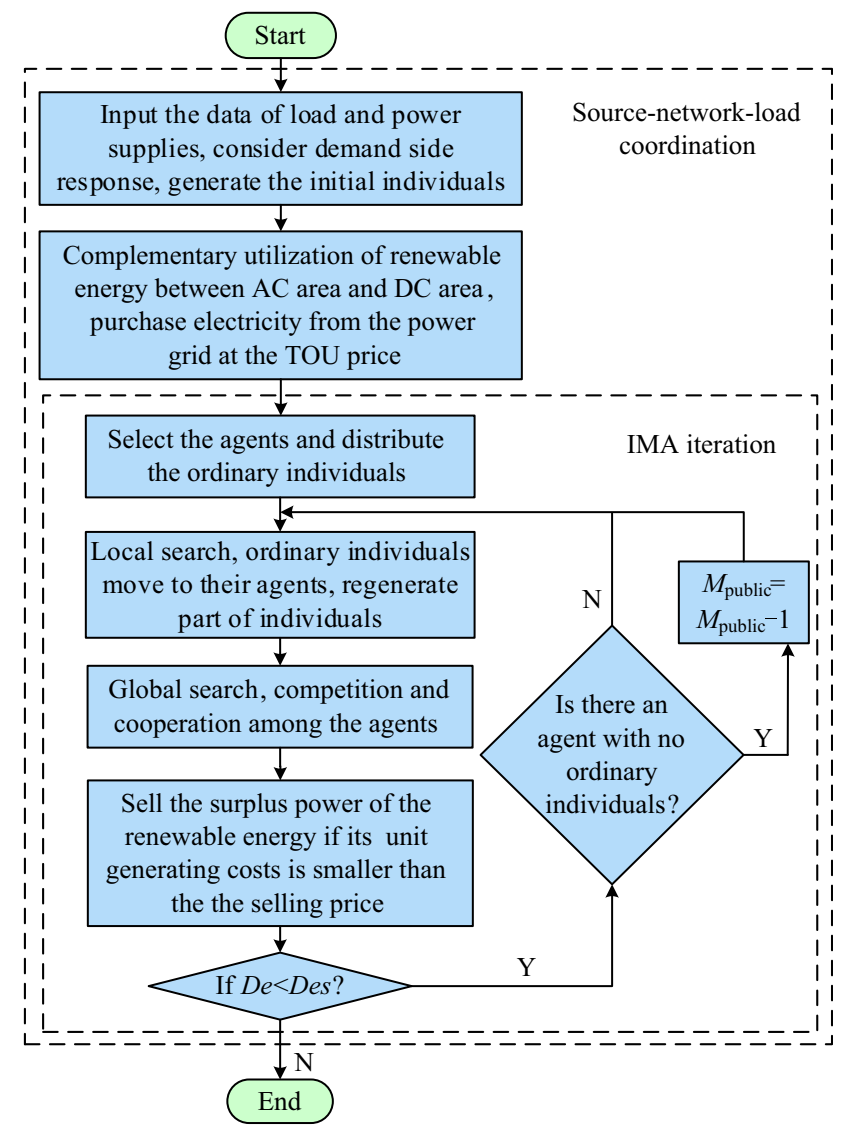

Fig. 5 Solution flow chart of the mathematical model

coefficients of each PS and cost coefficients of DEG are given in Appendix A Tables A2 and A3. The emission coefficients and emission factors of DEG are listed in Appendix A Table A4. Subsidy coefficient of shifting the load and unit generating subsidy price of renewable energy are respectively equal to $0.03 \$ / \mathrm{kWh}$ and $0.08 \$ / \mathrm{kWh}$. The initial value and the final value of SOC are 0.4 and its range is $0.1-0.9$. Figure 5 shows the IMA solution flow chart of the established mathematical model.

Figure 6 shows the load shifting situation in the load side. The first column and the second column of the Fig. 6 respectively show the load shifting situation of AC area and DC area. Figure $6 a, b$ shows the predictive data of the load and renewable energy, and the TOU price of the power grid, which is expressed as GP. Figure 6c, d show the load shifting situation for promoting the consumption rate of renewable energy. Figure $6 \mathrm{e}, \mathrm{f}$ shows the load shifting situation for reducing the running costs of the hybrid AC/DC microgrid. LAC and LDC represent the AC load curve and DC load curve. SLAC and SLDC respectively represent the shiftable load of AC load and DC load. FLAC and FLDC respectively represent the fixed load of AC load and DC load. From the Fig. 6c, it can be seen that the load is shifted-in when the output power of WT is 


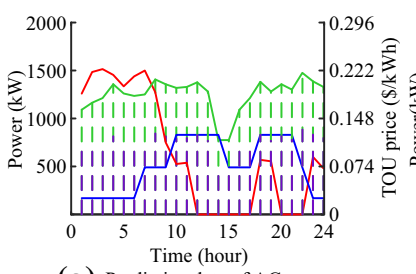

(a) Predictive data of $\mathrm{AC}$ area

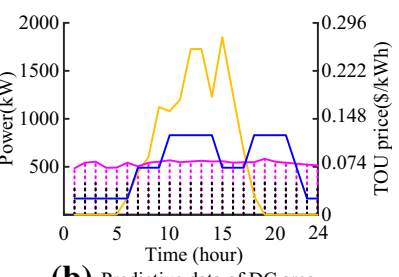

(b) Predictive data of DC area

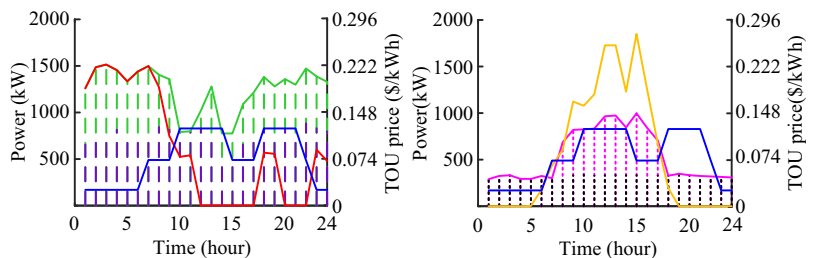

(c) Shift load for consuming WT in AC area (d) Shift load for consuming PV in DC area

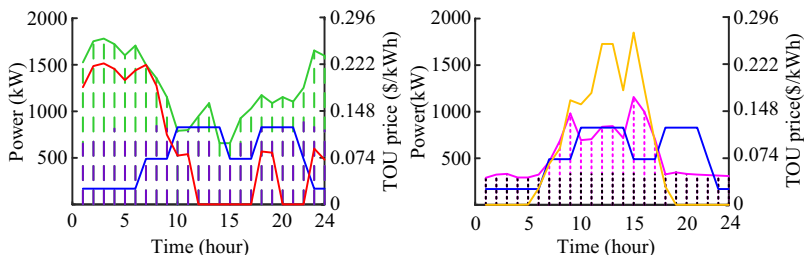

(e) Shift load for the costs of AC area

(f) Shift load for the costs of DC area

- WT; — TOU price of the grid; — PV; — Load in AC area — Load in DC area; - - Shiftable load in AC area; -....... Shiftable load in DC area - - Fixed load in AC area; ….... Fixed load in DC area

Fig. 6 Load shifting situation in the load side

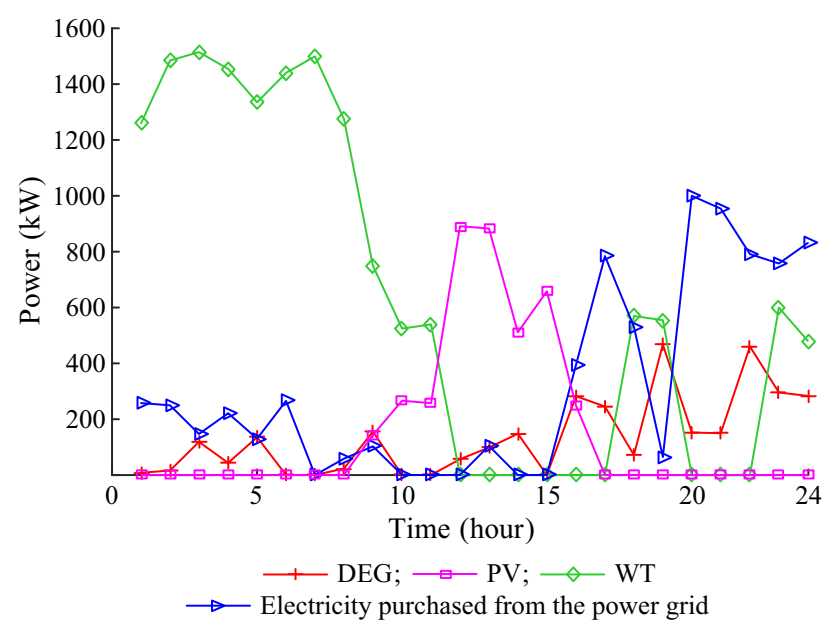

Fig. 7 Power output curves of AC area

bigger than the load value. Because of the peak output power of PV during the daytime, the DC load is shifted from night to day in the Fig. $6 \mathrm{~d}$ for promoting the consumption rate of PV. Considering the DSR based on price, the load shifting both occurs in AC area and DC area in the Fig. 6e, f to decrease the running costs of the hybrid $\mathrm{AC} /$ DC microgrid under the premise of guaranteeing the consumption rate of renewable energy.

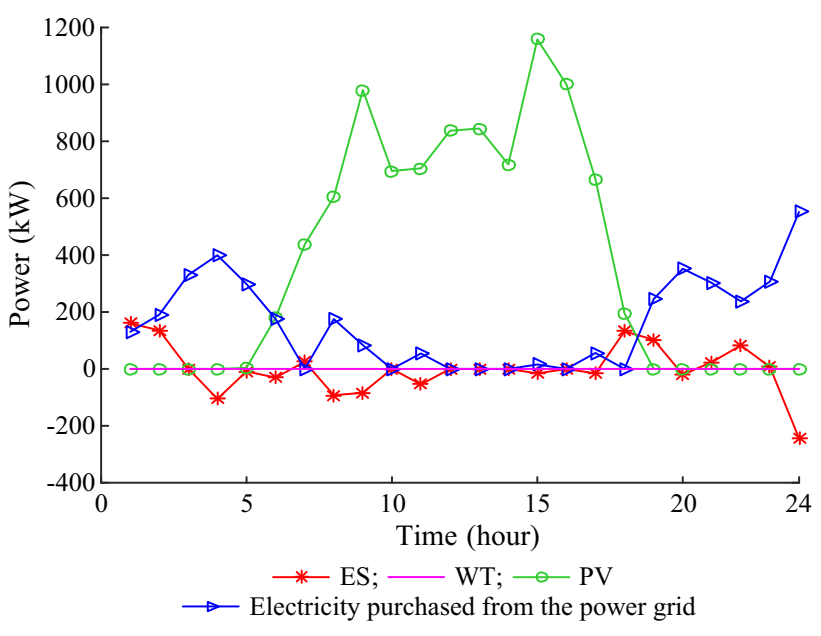

Fig. 8 Power output curves of DC area

According to the load shifting results, the output power of each power supply needs to be optimized through solving the optimal operation mathematical model of the hybrid AC/DC microgrid. The running time of the IMA is 10.22 s. Figure 7 shows the output power curves of each power supply in the AC area. Compared the power curves of the load and the renewable energy, it can be seen that the renewable energy generating costs is not considered and the power of renewable energy has the priority to supply the load, which effectively promotes the renewable energy consumption rate. PV is the power supply in DC area, which supplies the AC load. The complementary power supply between AC area and DC area is beneficial to the efficient utilization of renewable energy. Figure 8 shows the power supply curve of the DC area. WT is the power supply in the AC area, which is completely consumed in the AC area. There is no power of WT supplying in the DC area. According to Figs. 7 and 8, it indicates that both the $\mathrm{AC}$ area and the DC area try to purchase electricity during the valley price periods and the flat price periods and avoid purchasing electricity during the peak price periods, which can effectively reduce the operating costs of the hybrid $\mathrm{AC} /$ DC microgrid. AC area and DC area in the hybrid AC/DC microgrid achieve power flow through bidirectional power convertor (BPC). Figure 9 shows the power flowing through the BPCs. The power flow from AC to DC is positive, and the power flow from $\mathrm{DC}$ to $\mathrm{AC}$ is negative.

According to the above optimization results, the surplus power of the renewable energy during each period can be determined. Comparing the unit generating costs of the renewable energy and the selling price, the surplus power can be sold to the power grid if the former is smaller than the latter. The unit generating cost of renewable energy can be calculated from the cost functions in Section 2. The output power of renewable energy is replaced by $1 \mathrm{~kW}$ and supposing that the life span of PV and WT is 15 years. It can be 


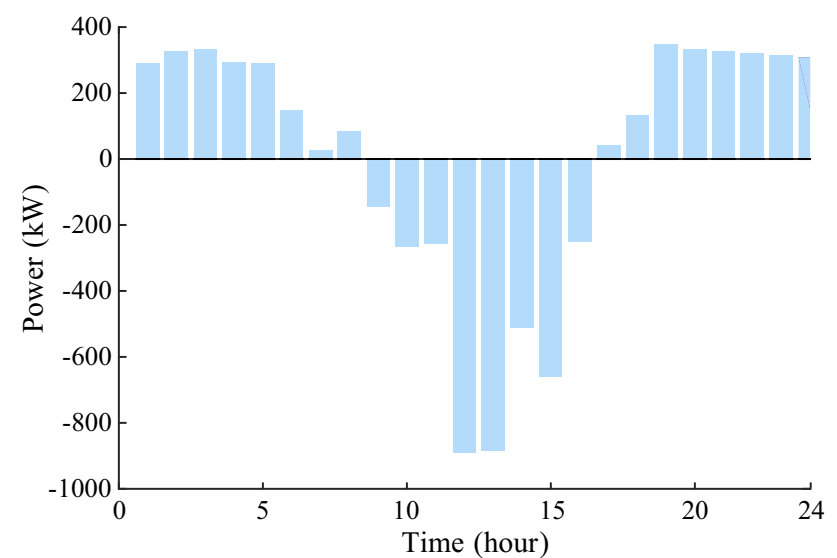

Fig. 9 Power flowing through the BPCs

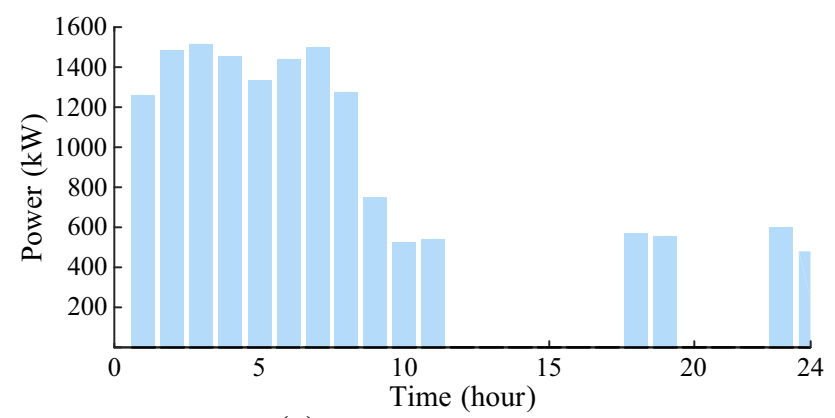

(a) Power flow situation of WT

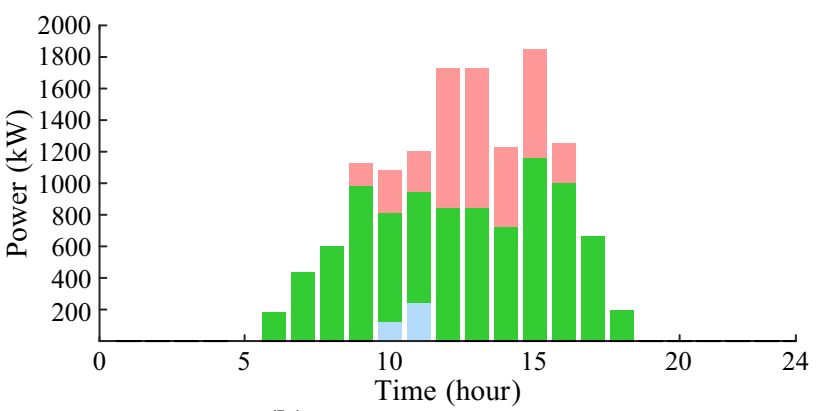

(b) Power flow situation of PV

Flow to AC area; Flow to DC area; Sold to power grid

Fig. 10 Power output situation of renewable energy

obtained that the unit generating cost of PV is $\$ 0.091$ and the unit generating cost of WT is $\$ 0.063$. The example data in this paper are from a demonstration project of hybrid $\mathrm{AC} /$ DC microgrid in a certain area of China. The price of surplus electricity is the same as that of local TOU price, which is listed in Appendix A Table A1. The power supplying situation of the renewable energy can be obtained as Fig. 10.

Figure 10a, b shows the power flow situation of WT and $\mathrm{PV}$, respectively. The load demand in the AC area is bigger than or equal to the power of WT at each time, so all output power of WT supplies the load in the AC area. There is no power of WT flowing to the DC area or sold to the power grid

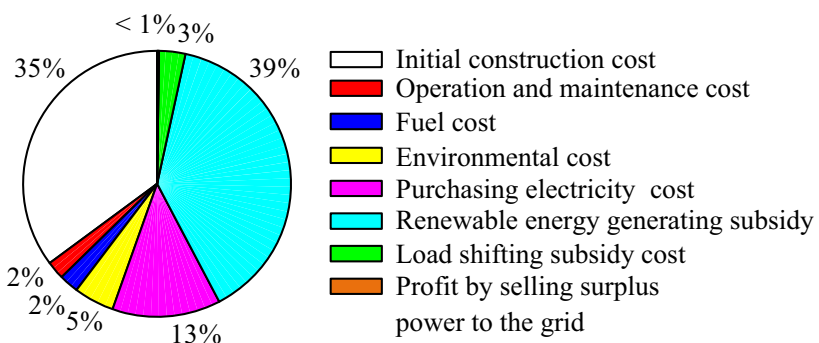

Fig. 11 Operation costs distributed situation

Table 3 Calculation results of each cost function

\begin{tabular}{lll}
\hline Calculation results & Mode A & Mode B \\
\hline Initial construction cost & $\$ 2334.2$ & $\$ 2321.9$ \\
Operation and maintenance cost & $\$ 145.2$ & $\$ 180.6$ \\
Fuel cost & $\$ 155.7$ & $\$ 186.5$ \\
Environmental cost & $\$ 323.5$ & $\$ 358.1$ \\
Purchasing electricity cost & $\$ 868.6$ & $\$ 904.2$ \\
Renewable energy generating subsidy & $\$ 2582.7$ & $\$ 2417.6$ \\
Load shifting subsidy cost & $\$ 211.5$ & 0 \\
Profits of selling the surplus power to the grid & $\$ 13.6$ & 0 \\
Total operating cost & $\$ 1442.4$ & $\$ 1533.7$ \\
Consumption rate & $100 \%$ & $82.14 \%$ \\
\hline
\end{tabular}

in Fig. 10a. From Fig. 10b, it is shown that the power of PV is difficult to be consumed completely in DC area because of its peak output power during the daytime. So there is part of output power of $\mathrm{PV}$ supplying the load in the $\mathrm{AC}$ area. When all load demand in the hybrid AC/DC microgrid is met, there is still the surplus power of PV. When the unit generation cost of $\mathrm{PV}$ is smaller than the selling price, the surplus power of PV is sold to the power grid, which not only improves the consumption rate of $\mathrm{PV}$, but also can reduce the operation cost of the hybrid AC/DC microgrid.

According to the optimization results, it can be calculated that the consumption rate of renewable energy is $100 \%$, and the total cost of the hybrid AC/DC microgrid is \$1442.4. Figure 11 is a diagram of the cost distribution situation of the hybrid AC/DC microgrid. From Fig. 11, it can be seen that the initial construction cost of the power supplies and the cost of the electricity from the power grid account for a large proportion of the total running cost of the hybrid $\mathrm{AC} /$ DC microgrid. But the amount of the renewable energy generation subsidies can significantly reduce the operation cost of the hybrid AC/DC microgrid.

In order to further prove the correctness and effectiveness of the multi-objective optimal operation method of hybrid AC/DC microgrid (Mode A), the IMA is applied to solve the mathematical model of the hybrid AC/DC microgrid without considering source-network-load 
coordination (Mode B) and the specific values of these cost functions are listed in Table 3. From Table 3, it can be seen that the total operation cost of Mode B is more than that of Mode A. Considering source-network-load coordination in Mode A, the consumption rate of renewable energy obviously increases and the environmental costs are reduced, which is conducive to environmental protection.

\section{Conclusion}

In this paper, the multi-objective optimal operation method of hybrid AC/DC microgrid considering sourcenetwork-load coordination is proposed to promote the consumption rate of renewable energy and decrease the operation cost of the hybrid AC/DC microgrid. The basic MA is improved and the excellent optimization performance of the IMA is proved through the test function. Compared with the basic MA, it can be seen that the IMA has a shorter running time and more ideal running results. Simulation results show that the incentive-based DSR and the price-based DSR both consider on the load side, the complementary utilization of renewable energy between $\mathrm{AC}$ area and DC area, and the electricity trading between the hybrid AC/DC microgrid and the big power grid are beneficial to improve the consumption rate of renewable energy and reduce the total running cost of the hybrid $\mathrm{AC} /$ DC microgrid. The convergence time of IMA is only $10.22 \mathrm{~s}$ and the consumption rate of renewable energy can reach $100 \%$. Comparing with the calculation results of hybrid AC/DC microgrid without considering source-network-load coordination, the cost of hybrid AC/DC microgrid considering source-network-load coordination is $\$ 1442.4$, which is reduced by $\$ 91.3$.

To sum up, it is necessary to consider the source-network-load coordination in the optimization operation of hybrid AC/DC microgrid, which is beneficial to the longterm development of renewable energy. Moreover, a great solving algorithm also has a significant influence on the optimal operation of hybrid AC/DC microgrid. It is the focus of future research to find the better modeling methods and solving methods.

Acknowledgements This work was supported by the National Natural Science Foundation of China (No. 51577068) and the National High Technology Research and Development Program of China (863 Program) (No. 2015AA050104).

Open Access This article is distributed under the terms of the Creative Commons Attribution 4.0 International License (http:// creativecommons.org/licenses/by/4.0/), which permits unrestricted use, distribution, and reproduction in any medium, provided you give appropriate credit to the original author(s) and the source, provide a link to the Creative Commons license, and indicate if changes were made.

\section{Appendix A}

See Tables A1, A2, A3 and A4.

Table A1 TOU price of the power grid

\begin{tabular}{ll}
\hline Time (hour) & Price $(\$ / \mathrm{kWh})$ \\
\hline $1-6,23-24$ & 0.025 \\
$7-9,15-17,22$ & 0.072 \\
$10-14,18-21$ & 0.122 \\
\hline
\end{tabular}

Table A2 Investment coefficient of each PS

\begin{tabular}{lc}
\hline PS & Investment coefficient \\
\hline PV & 36500 \\
WT & 23750 \\
ES & 1550 \\
\hline
\end{tabular}

Table A3 Fuel cost coefficients of DEG

\begin{tabular}{ll}
\hline Coefficient & Value \\
\hline$a$ & 6 \\
$b$ & 0.012 \\
$c$ & $8.5 \times 10^{-4}$ \\
\hline
\end{tabular}

Table A4 Emission coefficient and emission factor of DEG

\begin{tabular}{lll}
\hline Pollutant & Emission coefficient $(\$ / \mathrm{kg})$ & Emission factor $(\mathrm{kg} / \mathrm{kW})$ \\
\hline $\mathrm{NO}_{\mathbf{x}}$ & 4.340 & $2.18 \times 10^{-2}$ \\
$\mathrm{SO}_{2}$ & 1.023 & $4.54 \times 10^{-4}$ \\
$\mathrm{CO}_{2}$ & 0.014 & $1.40 \times 10^{-3}$ \\
\hline
\end{tabular}

\section{References}

[1] Saber AY, Venayagamoorthy GK (2011) Plug-in vehicles and renewable energy sources for cost and emission reductions. IEEE Trans Ind Electron 58(4):1229-1238

[2] Tummala ASLV, Inapakurthi R, Ramanarao PV (2018) Observer based sliding mode frequency control for multi-machine power systems with high renewable energy. J Mod Power Syst Clean Energy 6(3):473-481

[3] Wang L, Lam CS, Wong MC (2018) Analysis, control, and design of a hybrid grid-connected inverter for renewable energy generation with power quality conditioning. IEEE Trans Power Electron 33(8):6755-6768

[4] Rahbar K, Xu J, Zhang R (2015) Real-time energy storage management for renewable integration in microgrid: an off-line optimization approach. IEEE Trans Smart Grid 6(1):124-134

[5] Li P, Wang X, Lee WJ et al (2015) Dynamic power conditioning method of microgrid via adaptive inverse control. IEEE Trans Power Deliv 30(2):906-913 
[6] Fan M, Sun K, Lane D et al (2018) A novel generation rescheduling algorithm to improve power system reliability with high renewable energy penetration. IEEE Trans Power Syst 33(3):3349-3357

[7] Song X, Pan L, Zhang Z et al (2016) Source-network-load coordination planning study based on the big data from electric power metering. In: Proceedings of IEEE international conference on power and renewable energy, Shanghai, China, 21-23 October 2016, pp 339-344

[8] Wang F, Zhou L, Ren H et al (2018) Multi-objective optimization model of source-load-storage synergetic dispatch for a building energy management system based on TOU price demand response. IEEE Trans Ind Appl 54(2):1017-1028

[9] Geramifar H, Shahabi M, Barforoshi T (2017) Coordination of energy storage systems and DR resources for optimal scheduling of microgrids under uncertainties. IET Renew Power Gener 11(2):378-388

[10] Zhang Y, Wang R, Zhang $\mathrm{T}$ et al (2016) Model predictive control-based operation management for a residential microgrid with considering forecast uncertainties and demand response strategies. IET Gener Trans Distrib 10(10):2367-2378

[11] Zhu L, Zhou X, Zhang X et al (2018) Integrated resources planning in microgrids considering interruptible loads and shiftable loads. J Mod Power Syst Clean Energy 6(4):802-815

[12] Liu H, Zeng P, Guo J et al (2015) An optimization strategy of controlled electric vehicle charging considering demand side response and regional wind and photovoltaic. J Mod Power Syst Clean Energy 3(2):232-239

[13] Liu N, He L, Yu X et al (2018) Multiparty energy management for grid-connected microgrids with heat- and electricity-coupled demand response. IEEE Trans Ind Inf 14(5):1887-1897

[14] Daraeepour A, Kazempour SJ, Patiño-Echeverri D et al (2016) Strategic demand-side response to wind power integration. IEEE Trans Power Syst 31(5):3495-3505

[15] Lasseter RH (2011) Smart distribution: coupled microgrids. Proc IEEE 99(6):1074-1082

[16] Krismanto AU, Mithulananthan N, Kamwa I (2018) Oscillatory stability assessment of microgrid in autonomous operation with uncertainties. IET Renew Power Gener 12(4):494-504

[17] Chaouachi A, Kamel RM, Andoulsi R et al (2013) Multiobjective intelligent energy management for a microgrid. IEEE Trans Ind Electron 60(4):1688-1699

[18] Wang Z, Chen Y, Mei S et al (2017) Optimal expansion planning of isolated microgrid with renewable energy resources and controllable loads. IET Renew Power Gener 11(7):931-940

[19] Minchala-Avila LI, Garza-Castañón L, Zhang Y et al (2016) Optimal energy management for stable operation of an islanded microgrid. IEEE Trans Ind Inform 12(4):1361-1370

[20] Li J, Liu Y, Wu L (2018) Optimal operation for communitybased multi-party microgrid in grid-connected and islanded modes. IEEE Trans Smart Grid 9(2):756-765

[21] Wu X, Wang X, Qu C (2014) A hierarchical framework for generation scheduling of microgrids. IEEE Trans Power Deliv 29(6):2448-2457

[22] Li P, Xu D, Zhou Z et al (2016) Stochastic optimal operation of microgrid based on chaotic binary particle swarm optimization. IEEE Trans Smart Grid 7(1):66-73

[23] Zare M, Niknam T, Azizipanah-Abarghooee R et al (2016) New stochastic bi-objective optimal cost and chance of operation management approach for smart microgrid. IEEE Trans Ind Inform 12(6):2031-2040

[24] Hao R, Jiang Z, Ai Q et al (2018) Hierarchical optimization strategy in microgrid based on the consensus of multi-agent system. IET Gener Transm Distrib 12(10):2444-2451

[25] Eghtedarpour N, Farjah E (2014) Power control and management in a hybrid AC/DC microgrid. IEEE Trans Smart Grid 5(3): 1494-1505

[26] Liu X, Wang P, Loh PC (2011) A hybrid ac/dc microgrid and its coordination control. IEEE Trans Smart Grid 2(2):278-286

[27] Ding GQ, Gao F, Zhang S et al (2014) Control of hybrid AC/DC microgrid under islanding operational conditions. J Mod Power Syst Clean Energy 2(3):223-232

[28] Hasanien HM (2018) Whale optimisation algorithm for automatic generation control of interconnected modern power systems including renewable energy sources. IET Gener Transm Distrib 12(3):607-614

[29] Marzband M, Azarinejadian F, Savaghebi M et al (2017) An optimal energy management system for islanded microgrids based on multiperiod artificial bee colony combined with markov chain. IEEE Syst J 11(3):1712-1722

[30] Sharma S, Bhattacharjee S, Bhattacharya A (2016) Grey wolf optimisation for optimal sizing of battery energy storage device to minimise operation cost of microgrid. IET Gener Transm Distrib 10(3):625-637

[31] Bahrami S, Wong VWS (2018) Security-constrained unit commitment for AC-DC grids With generation and load uncertainty. IEEE Trans Power Syst 33(3):2717-2732

[32] Amini TMH, Boroojeni KG, Dragičević T et al (2017) A comprehensive cloud-based real-time simulation framework for oblivious power routing in clusters of DC microgrids. In: Proceedings of IEEE 2nd international conference on DC microgrids, Nuremburg, Germany, 27-29 June 2017, pp 270-273

[33] Moscato P (1989) On evolution, search, optimization, genetic algorithms and martial arts: towards memetic algorithm. California Institute of Technology, Pasadena

[34] Yoshida H, Kawata K, Fukuyama Y et al (2000) A particle swarm optimization for reactive power and voltage control considering voltage security assessment. IEEE Trans Power Syst 15(4):1232-1239

Peng LI received the B.S., M.S. and Ph.D. degrees from the North China Electric Power University, China, in 1988, 1993 and 2004, respectively, all in electrical engineering. He is now a Full Professor with the North China Electric Power University, Baoding, China. His research interests include distributed generation, microgrid, power quality analysis and control, power electronics technology application in power system, etc. He is a Senior Member of the IEEE.

Miaomiao ZHENG received the B.S. degree in electrical engineering from Ningxia University, Yinchuan, China, in 2016. She is currently pursuing the post-graduate degree with the School of Electrical and Electronic Engineering, North China Electric Power University, Baoding, China. Her current research interests include distributed generation and microgrid technology. 